\title{
Mechanical properties of betel nut and glass fibre reinforced hybrid polyethylene composites
}

\author{
M. Merajul Haque* and Mahbub Hasan \\ Department of Materials and Metallurgical Engineering \\ Bangladesh University of Engineering and Technology, Dhaka-1000, Bangladesh \\ *E-mail: merajulh34@gmail.com \\ Phone: +8801916861734.
}

\begin{abstract}
This paper describes an investigation done on the mechanical properties of betel nut-glass fibre reinforced hybrid polyethylene composites by varying the fibre loading and ratio. By using the hot press method with four levels of fibre loading (5, 10, 15 and $20 \mathrm{wt} \%$ ), different composites were manufactured. For the $20 \mathrm{wt} \%$ fibre-loaded composites, the fibre ratio was also varied (betel nut:glass $=1: 1,3: 1$ and 1:3). Fourier transform infrared spectroscopic (FTIR) analysis and tensile, flexural and hardness tests were conducted for the characterisation of the composites. The FTIR analysis indicated the presence of hemicelluloses, lignin and moisture content in the prepared composites, which are liable to decrease tensile strength. As a result, the tensile strength decreased but Young's modulus increased with fibre loading. On the other hand, flexural strength, flexural modulus and hardness increased with fibre loading. SEM analysis revealed the morphological characteristics of the fibre-polyethylene matrix. Above all, the composite containing $20 \mathrm{wt} \%$ fibre at a 1:3 ratio of betel nut and glass showed the highest mechanical properties (tensile, flexural and hardness).
\end{abstract}

Keywords: Glass fibre; betel nut; polyethylene; hybrid composite; properties.

\section{INTRODUCTION}

Hybrid fibre-polymer composites provide designers with the freedom to tailor composites and achieve properties in a more cost-effective way that cannot be attained in binary systems containing one type of fibre/filler dispersed in the matrix. In addition, the advantages of one type of fibre could complement what is lacking in the other. As a result, a balance in performance and cost could be achieved through proper material design. Hybridisation of a natural fibre with a stronger synthetic or natural fibre can also improve the mechanical strength and other properties of the composite. The automotive industry is the main client for natural fibre usage because of the useful properties of natural fibres [1]. Composites can be used in producing mirror casings, doors, windows and various parts of car interiors, together with truck cabins, various panels, shelves, trim parts and brake shoes, which are attractive for the automotive industry. The betel nut is the fruit of areca palm tree (Areca catechu), a species of palm that is available and grows widely across Asia [2]. Bangladesh contributes to the global production of betel nut. In Bangladesh, betel nut fibres are traditionally used for housing insulation material [1]. Betel nut fibres are very cheap. The major drawback of utilising natural fibre composites is the incompatibility between the hydrophilic nature of natural fibres and the 
hydrophobic properties of the polymer matrix, which generally leads to poor fibre-matrix interfacial adhesion [3].

Glass fibres are mostly used as reinforcement for polymeric matrix composites. Their main advantages are the relationship between their low cost, high tensile strength, high chemical resistance, and insulating properties. E-glass and S-glass are the types of fibres most commonly used in the fibre-reinforced plastic industry. E-glass fibres have the lowest cost of commercially available reinforcing glass fibres, which is the reason for their widespread use in the fibre-reinforced plastic industry [4].Suitable compositions and orientations of fibres can provide desired properties, with the functional characteristics of these composites equal to steel, a higher stiffness than aluminium and the specific gravity was one-quarter of that of steel. Glass fibre-reinforced composite materials are used in the marine, automotive and piping industries because of good environmental resistance, better damage tolerance for impact loading, and high specific strength and stiffness. The high cost of polymers was a limiting factor in their use for commercial applications, although the use of fillers improved the properties of composites and ultimately reduced the cost of the preparation and the product [5]. The huge applications of polymers in almost all fields ranging from sophisticated articles such as prosthetic hip and knee joints to disposable food utensils implies their significance and importance in our everyday life. Polyethylene is the most widely used linear hydrocarbon polymer with applications in food packaging, textiles, lab equipment and automotive components. Polyethylene is totally linear and is available with varying densities from 0.91 to $0.97 \mathrm{~g} / \mathrm{cc}$ [5]. A great deal of research has been carried out on natural fibres hybridised with synthetic fibres. However, research with betel nut and glass fibres in the presence of a polyethylene matrix has not yet been studied. Thus, this paper will provide some valuable insight into the hybridisation of betel nut and glass fibres with polyethylene. Polyethylene was chosen as the matrix material owing to its low cost. This paper discusses a cost-effective method to produce eco-friendly hybrid composites using natural fibres. The paper specifically looks at the use of betel nut fibres with glass and polyethylene.

\section{METHODS AND MATERIALS}

\section{Materials}

Commercial grade polyethylene, betel nut and glass fibre were used in this study. All of them were collected from the local market. The polyethylene was LDPE in granular form with a melting point of $140{ }^{\circ} \mathrm{C}$ and white in colour. Betel nut fruits were soaked in water for 10 days at room temperature to loosen the fibre. Afterwards, betel nut fibres were separated from the nut part by hand.

\section{Manufacturing of Composites}

Hybrid composites of polyethylene matrix with varying amounts of betel nut and glass fibre were manufactured by hot press technique. The dimension of the aluminium die was $150 \times 150 \times 5 \mathrm{~mm}$. A hydraulic type machine was used with a maximum load of $35 \mathrm{kN}$ and a maximum temperature of $300^{\circ} \mathrm{C}$. The fibre loading was varied at 5, 10, 15 and 20 wt. \% with the ratio of betel nut and glass as 1:1. Composites were also prepared for betel nut to glass ratios of 3:1 and 1:3 for $20 \mathrm{wt} \%$ fibre loading. Fibres of 3-5 mm were used. First, the required amount of fibre and polyethylene were weighed in a balance. The premixed mixture was placed inside the die. Pressure of $30 \mathrm{kN}$ was applied to press the fibre-matrix mixture inside the die. The temperature was initially raised to $140{ }^{\circ} \mathrm{C}$ and held there for around 18-20 minutes then temperature was raised to $160{ }^{\circ} \mathrm{C}$. This fact 
depends on the thickness requirement. The die was then cooled to room temperature. Composites were withdrawn from the die by releasing the pressure. All the composites were manufactured using this procedure.

\section{FTIR Spectroscopy}

In this research, FTIR spectra of betel nut-glass fibre reinforced hybrid composites were recorded on a Nicolet 380 spectrophotometer with co-addition of 32 scans. First powdered samples were collected by scratching the composite with a knife. Then potassium bromide $(\mathrm{KBr})$ was mixed (at a ratio KBr:sample $=100: 1)$ with the samples in a mortar pestle, which acts as a reagent. The mixture was then placed in a die of $13 \mathrm{~mm}$ diameter and pressed (mechanical presser at 8 tons of pressure) to form a pellet, which was then placed on the sample holder to carry out the spectroscopy.

\section{Mechanical Testing}

Tensile, flexural and hardness tests were carried out. In each case, five samples were tested and average values were reported. The tensile test was conducted using an Instron UTM machine at a crosshead speed of $4 \mathrm{~mm} / \mathrm{min}$ (system Id 3369J8567, maximum capacity $50 \mathrm{kN}$ ) according to ASTM D 638-01 (In: Annual book of ASTM standard, 2002[6]). Static flexural tests were carried out according to ASTM D 790-00 (Annual book of ASTM standards, 2002[7]) using the same testing machine. The hardness of the composite was measured using a Durometer hardness tester in Shore (A) scale.

\section{ScanningElectron Microscopy}

The tensile fracture surface morphology of the betel nut-glass fibre reinforced hybrid polyethylene composites was viewed using a field emission scanning electron microscope JEOL FESEM analyser (model no JSM-7600F). Interfacial bonding between the fibre and the polyethylene matrix was also viewed from the perpendicular direction to the fractured surfaces.

\section{RESULTS AND DISCUSSION}

\section{FTIR Spectroscopic Analysis}

The FTIR spectrum of polyethylene is shown in Figure 1. In the spectrum, the major peaks found at $2923 \mathrm{~cm}^{-1}, 2852 \mathrm{~cm}^{-1}, 1464 \mathrm{~cm}^{-1}, 1367 \mathrm{~cm}^{-1}$ and $1631 \mathrm{~cm}^{-1}$ correspond respectively to the strong $\mathrm{CH}_{2}$ asymmetric stretch, $\mathrm{CH}_{2}$ (methylene) symmetric strong stretch, strong bending deformation, medium wagging deformation and presence of water vapour in air. Another important peak at $719 \mathrm{~cm}^{-1}$ corresponds to the medium rocking deformation [6]. The FTIR spectrum of the prepared composite shows an absorption peak around $3477 \mathrm{~cm}^{-1}$, corresponding to the presence of an $-\mathrm{OH}$ group and other peaks at $1633 \mathrm{~cm}^{-1}, 1373 \mathrm{~cm}^{-1}$ and $1107 \mathrm{~cm}^{-1}$ corresponding to aromatic $\mathrm{C}=\mathrm{C}$ in-plane alkenes (lignin) and the $\mathrm{C}-\mathrm{H}$ bending bond structure of cellulose, hemicelluloses, lignin and glass fibre, respectively as shown in Figure 2 [9].

\section{Tensile and flexural strength}

The tensile strength was measured for the composites containing 5, 10, 15 and $20 \mathrm{wt} \%$ fibre. It was observed that with increasing the fibre loading, the tensile strength decreases. Initially it was increased for 5\% fibre loading, but as the fibre loading further increases, 
contact between the fibre and the matrix also increases to make the interfacial bonding weaker, which gradually reduced the tensile strength [7-10], as shown in Figure 4.

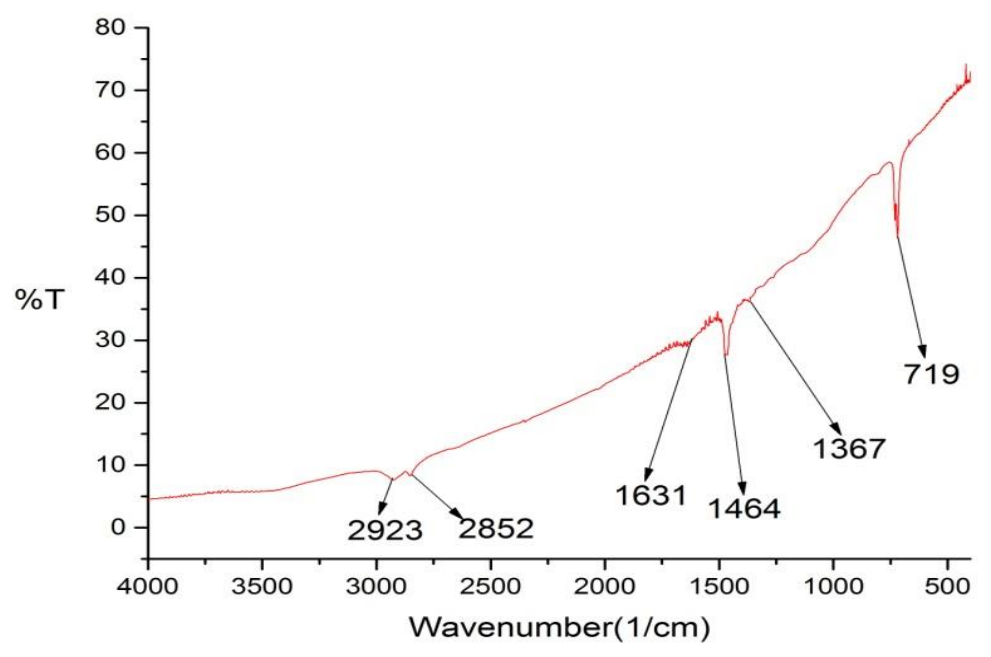

Figure 1. FTIR spectrum of polyethylene.

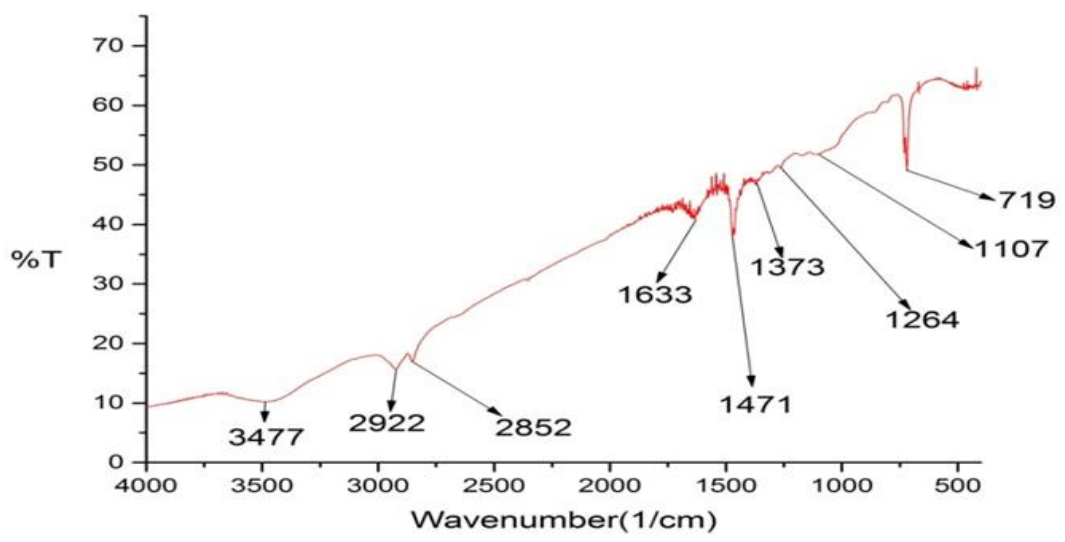

Figure 2. FTIR spectrum of betel nut:glass (1:3) $20 \mathrm{wt} \%$ fibre reinforced composite.

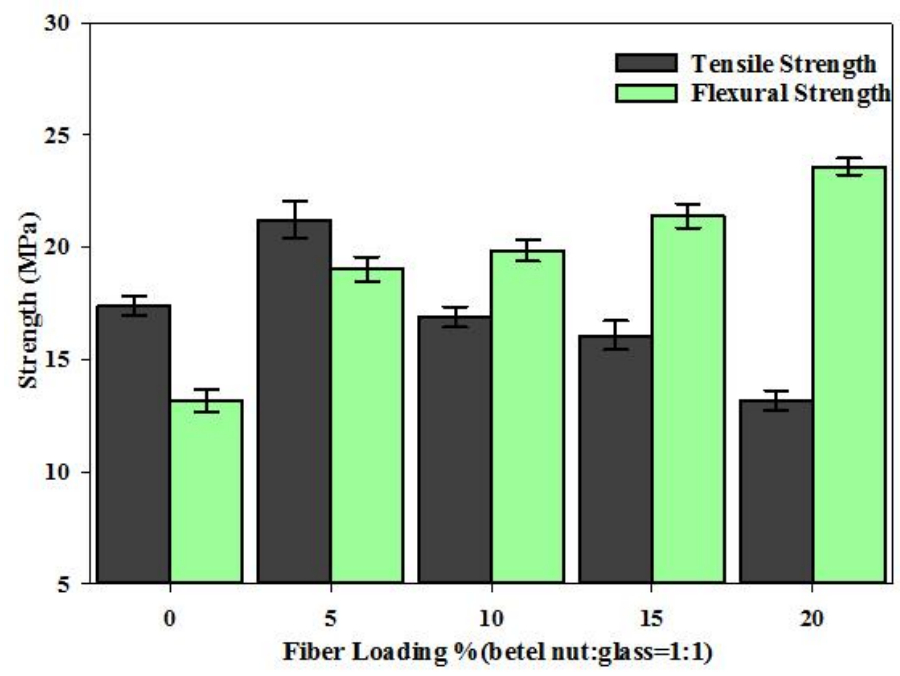

Figure 4. Tensile and flexural strength variations with different fibre loadings. 
The flexural strength was also measured for the composites containing 5, 10, 15 and $20 \mathrm{wt} \%$ fibre. These results reveals that the flexural strength increased with fibre loading. Owing to the suitable alignment of the polyethylene chain with the fibre, weak fibre-matrix adhesion might be easily overcome. Therefore, as fibre loading increases [10], the possibility of strong fibre-matrix adhesion increases, which results is enhanced flexural strength [8], as shown in Figure 4.

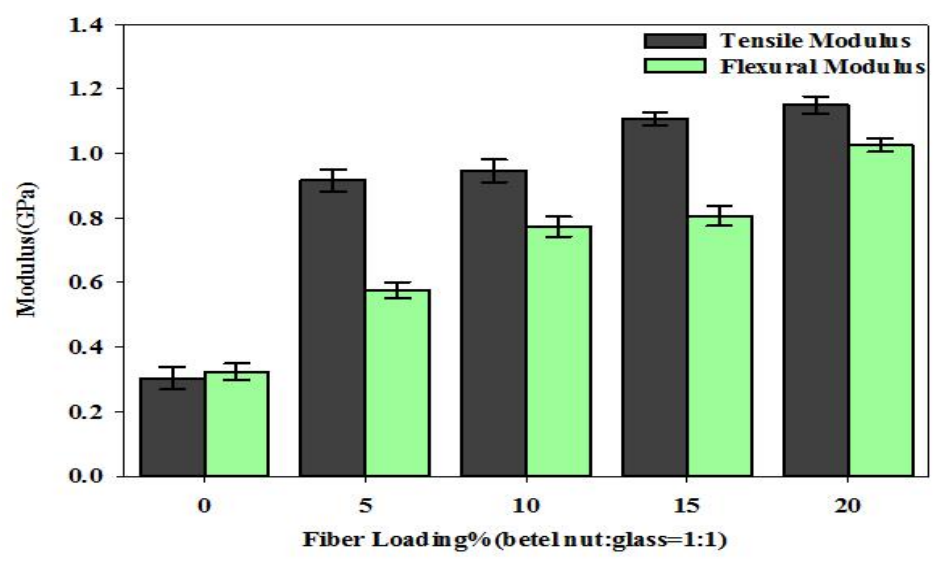

Figure 5. Tensile and flexural modulus variations with different fibre loadings.

The Young's modulus and flexural modulus were measured for the composites containing 5, 10, 15 and $20 \mathrm{wt} \%$ fibre, as shown in Figure 5. Here, Young's modulus increased with an increase in fibre loading [11]. When the interfacial bonding becomes poor or weak, it creates separated micro-spaces that prevent stress propagation between the fibre and matrix [10]. This obstruction for stress propagation enhances as the fibre loading increases and it causes the stiffness to increase. Furthermore, incorporation of fibre into the polymer matrix reduced the matrix mobility, which in turn also enhances the stiffness [12]. Betel nut (1.381 GPa) and glass (72.3 GPa) are high modulus materials. Higher fibre concentrations demand higher stress for equal deformation [9]. The results shows that flexural modulus increased with fibre loading. Since both areca and glass are high modulus materials, higher fibre concentrations demand higher stress for the same deformation and increased fibre-matrix adhesion provides for increased stress transfer from the matrix to the fibre. Thus this result can be attributed to the incorporation of rigid glass fibre into the soft PE matrix. As a result, high modulus fibre incorporation into the soft polyethylene matrix increased the flexural modulus.

\section{Hardness Analysis}

It imparts lower hardness, such as raw polyethylene when the matrix becomes more flexible. The hardness of a composite also depends on the distribution of the fibre into the matrix. For instance, in this research raw polyethylene's hardness of around 70 increases to above 90 as the fibre content increased above $15 \mathrm{wt} . \%$. Better dispersion of the fibre into the matrix with minimisation of voids between the matrix and the fibre enhanced this hardness. Thus, more fibre incorporation into polyethylene makes it harder. The hardness variation with different fibre loading [13] is shown in Figure 6. 


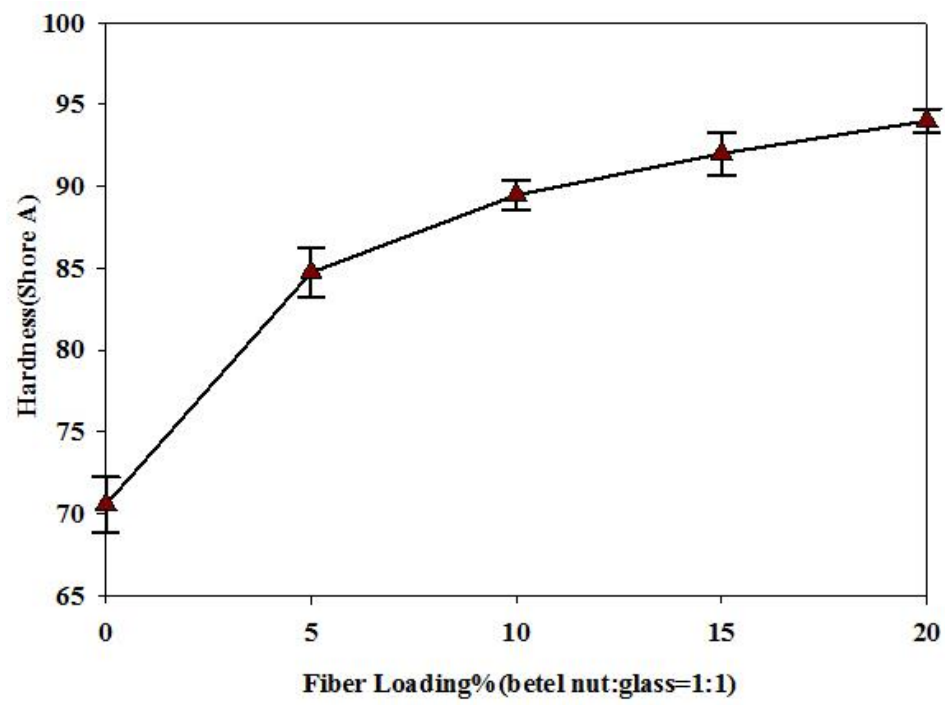

Figure 6. Hardness (shore A) variation at different fibre loadings.

\section{Effect of Fibre Ratio}

The variation of the mechanical properties of the composites reinforced with different ratios of betel nut and glass fibre are shown in Figures 7-9. Both the tensile strength and flexural strength increased with increasing glass fibre loading. The chemical composition of the fibre and its internal structure affect the tensile strength [11]. As a result, highstrength glass fibre (2.0 GPa) compared with betel nut (166.03 MPa) enhanced the tensile strength when the ratio of betel nut and glass is 1:3, as shown in Figure 7. In a three-point flexure test, failure occurred owing to bending and shearing. The increased flexural strength of the hybrid composites with the loading of glass fibre was mainly owing to the increased resistance to shearing of the composites as a result of the inclusion of rigid glass fibre [10].

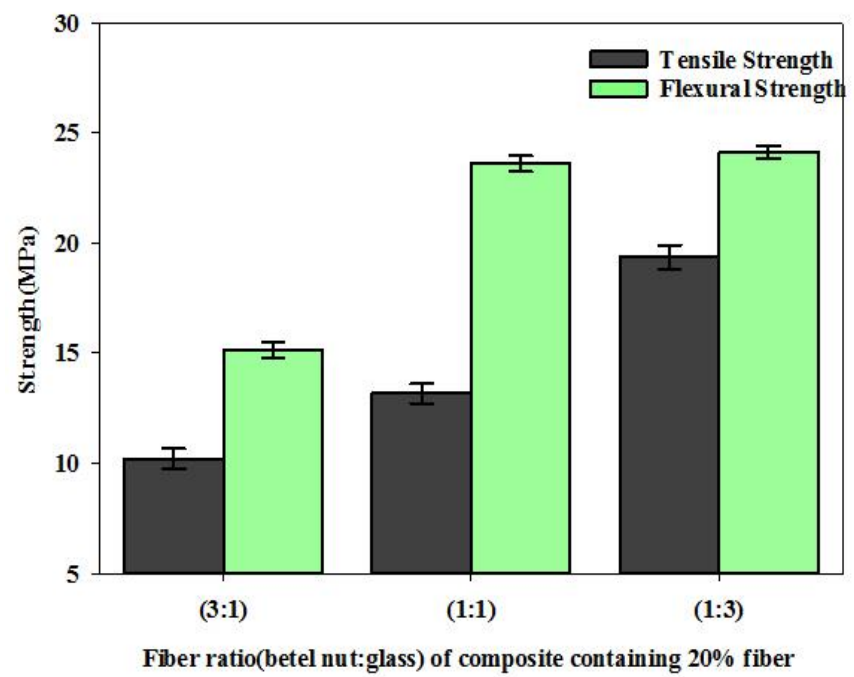

Figure 7. Tensile and flexural strength variation at different fibre ratios for $20 \mathrm{wt} \%$ fibre loading. 
It can be seen that the tensile and flexural modulus increased with increasing glass fibre percentage, as shown in Figure 8. A higher concentration of high modulus fibre demands more stress for equal deformation. Therefore, it increases the tensile and flexural modulus of the composite. As glass fibre has a higher modulus $(72.3 \mathrm{GPa})$ than betel nut $(1.381 \mathrm{GPa})$, a greater proportion of glass fibre increases the modulus. As a result, with the enhancement of the glass fibre ratio from 1 to 3, the flexural modulus increased from 0.42 to $1.17 \mathrm{GPa}$. With the change of the rigid glass fibre ratio from 1 to 3 , hardness also increased from 90.23 to 96.7 (shore A), as shown in Figure 9.

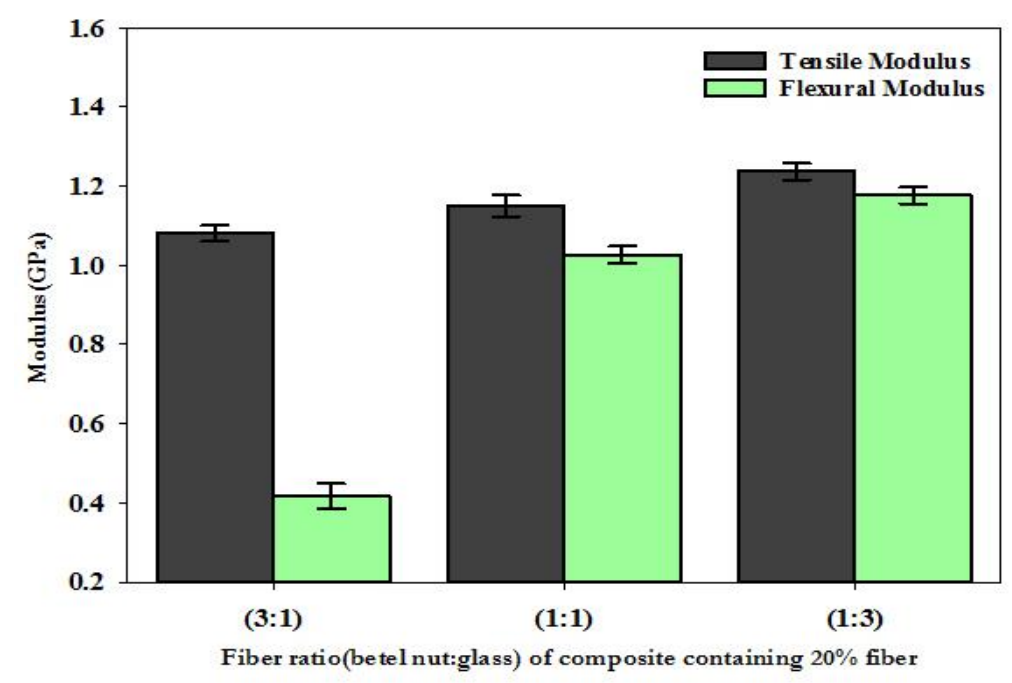

Figure 8 . Tensile and flexural modulus variation at different fibre ratios for $20 \%$ fibre loading.

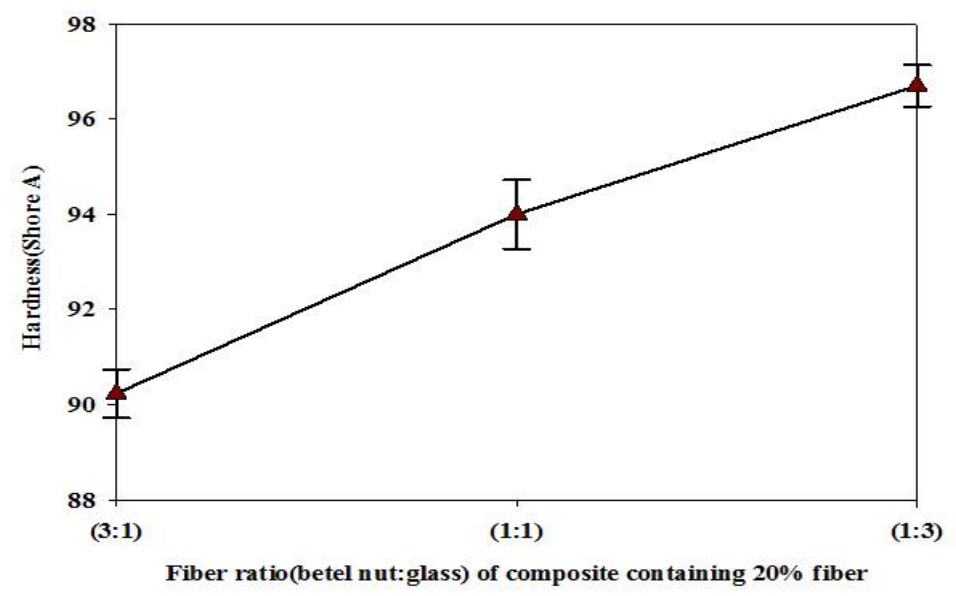

Figure 9. Hardness (shore A) variation at different fibre ratios for $20 \mathrm{wt} \%$ fibre loading.

\section{Surface Morphology}

The SEM images of the tensile specimen fracture surfaces are shown in Figure 10. The fracture surface of the composites containing $20 \mathrm{wt} \%$ fibre with a betel nut and glass fibre ratio of 1:1 shows numerous voids with fibre agglomeration in Figure 10(a). This indicates that interfacial bonding between the fibre and polymer matrix was very poor, and therefore tensile strength decreased [14]. The composite containing $20 \mathrm{wt} \%$ fibre with a betel nut and glass fibre ratio of 1:3 showed uniform dispersion of the fibres into the 
matrix and good bonding characteristics in Figure 10(b). As a result, higher tensile strength was found in this composite. However, the composite containing $20 \mathrm{wt} \%$ fibre with a betel nut and glass fibre ratio of 3:1 shown in Figure 10(c) indicates fibre pull-out, deboning and fibre agglomeration, which reduced the tensile strength. This increased weak interfacial area between the fibre and the matrix was not desirable.
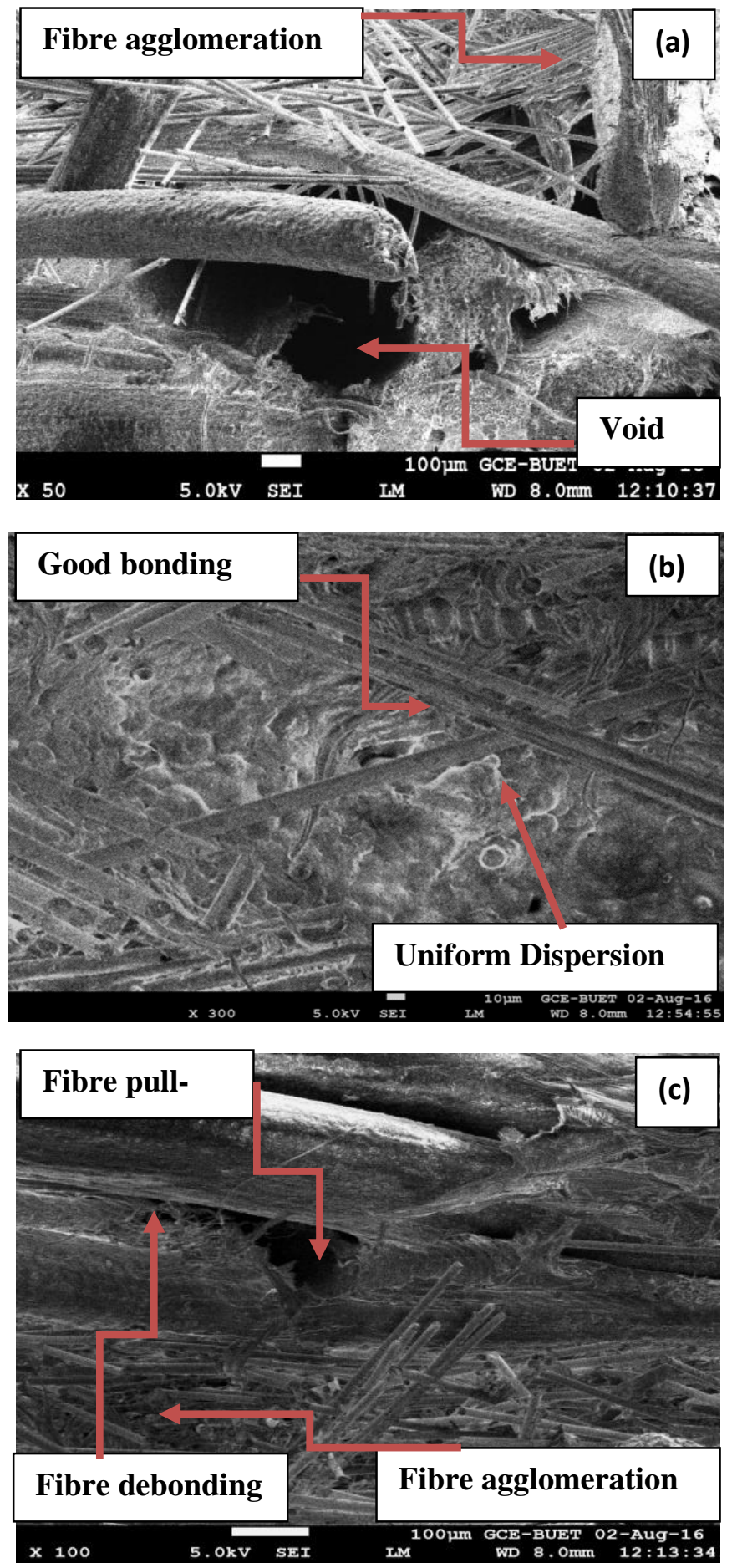

Figure 10. SEM of tensile fracture surface of $20 \mathrm{wt} \%$ fibre loaded composites with betel nut:glass ratios of: (a) 1:1, (b) 1:3 and (c) 3:1. 


\section{CONCLUSIONS}

The present study reveals that betel nut and glass fibre could be successfully used as reinforcing fibres in polyethylene composites. Based on the experimental results and analysis, the following conclusions have been drawn:

i) FTIR spectroscopic analysis of the prepared composites showed the presence of hemicelluloses, lignin and $-\mathrm{OH}$ groups of betel nut fibres, which hamper good interfacial bonding.

ii) The tensile strength of the prepared composites decreased as the fibre loading increased, but the Young's modulus increased. Maximum tensile strength and Young's modulus of 19.36 MPa and 1.23 GPa, respectively, were found.

iii) Maximum flexural strength (24.13 MPa), Maximum flexural modulus (1.17 GPa); changing the glass fibre ratio from 1 to 3 results it.

iv) The maximum hardness of the composite was found to be 96.7 (shore A) whereas polyethylene has only around 70 (shore A).

v) For the composite containing betel nut and glass fibre at a 1:3 ratio, the interfacial fibre-polyethylene interaction was good. Uniform dispersion of fibre was observed, which was confirmed by SEM results.

vi) Finally, these polyethylene-based composites can be used successfully in the automotive and food packaging industries.

Above all, the composite containing $20 \mathrm{wt} \%$ fibre at a 1:3 ratio of betel nut and glass exhibited the highest mechanical properties (tensile, flexural and hardness).

\section{ACKNOWLEDGEMENT}

The authors would like to thank Bangladesh University of Engineering and Technology (BUET), Dhaka, Bangladesh for their financial support during the present research.

\section{REFERENCES}

[1] Yusriah L, Sapuan S, Zainudin E, Mariatti M. Exploring the potential of betel nut husk fiber as reinforcement in polymer composites: Effect of fiber maturity. Procedia Chemistry. 2012;4:87-94.

[2] Islam MS, Ahmad MB, Hasan M, Aziz SA, Jawaid M, Haafiz MM, et al. Natural fiber-reinforced hybrid polymer nanocomposites: effect of fiber mixing and nanoclay on physical, mechanical, and biodegradable properties. BioResources. 2015;10:1394-407.

[3] Etcheverry M, Barbosa SE. Glass fiber reinforced polypropylene mechanical properties enhancement by adhesion improvement. Materials. 2012;5:1084-113.

[4] Sathishkumar T, Satheeshkumar S, Naveen J. Glass fiber-reinforced polymer composites-a review. Journal of Reinforced Plastics and Composites. 2014;33:1258-75.

[5] Arutchelvi J, Sudhakar M, Arkatkar A, Doble M, Bhaduri S, Uppara PV. Biodegradation of polyethylene and polypropylene. Indian Journal of Biotechnology. 2008;7:9.

[6] Petersen HN, Kusano Y, Brøndsted P, Almdal K. Preliminary characterization of glass fiber sizing. 34th Risø International Symposium on Materials Science2013. p. 333-40. 
[7] Jamil MS, Ahmad I, Abdullah I. Effects of rice husk filler on the mechanical and thermal properties of liquid natural rubber compatibilized high-density polyethylene/natural rubber blends. Journal of Polymer Research. 2006;13:31521.

[8] Rahman MR, Huque MM, Islam MN, Hasan M. Improvement of physicomechanical properties of jute fiber reinforced polypropylene composites by posttreatment. Composites Part A: Applied Science and Manufacturing. 2008;39:1739-47.

[9] Rahman MR, Huque MM, Islam MN, Hasan M. Mechanical properties of polypropylene composites reinforced with chemically treated abaca. Composites Part A: Applied Science and Manufacturing. 2009;40:511-7.

[10] Yang H-S, Kim H-J, Park H-J, Lee B-J, Hwang T-S. Water absorption behavior and mechanical properties of lignocellulosic filler-polyolefin bio-composites. Composite Structures. 2006;72:429-37.

[11] Hatta M, Nasrul M, Akashah NA. Mechanical properties of polystyrene/polypropylene reinforced coconut and jute fibers. 2008.

[12] Mir SS, Nafsin N, Hasan M, Hasan N, Hassan A. Improvement of physicomechanical properties of coir-polypropylene biocomposites by fiber chemical treatment. Materials \& Design. 2013;52:251-7.

[13] Siddika S, Mansura F, Hasan M, Hassan A. Effect of reinforcement and chemical treatment of fiber on The Properties of jute-coir fiber reinforced hybrid polypropylene composites. Fibers and Polymers. 2014;15:1023-8.

[14] Islam MS, Hasbullah NAB, Hasan M, Talib ZA, Jawaid M, Haafiz MM. Physical, mechanical and biodegradable properties of kenaf/coir hybrid fiber reinforced polymer nanocomposites. Materials Today Communications. 2015;4:69-76. 